\title{
Critical Care in Patient with Neuromuscular Cervicothoracic Kyphosis
}

\author{
Kwang-Ui Hong, Jong-myung Jung, Seung-Jae Hyun, Ki-Jeong Kim, Tae-Ahn Jahng \\ Department of Neurosurgery, Spine Center, Seoul National University Bundang Hospital, Seoul National University College of Medicine, Seongnam, Korea
}

Received: August 9, 2019

Accepted: August 28, 2019

Corresponding Author:

Seung-Jae Hyun, M.D., Ph.D.

Department of Neurosurgery, Spine Center, Seoul National University Bundang Hospital, Seoul National University College of Medicine, 82 Gumi-ro

173beon-gil, Bundang-gu, Seongnam 13620, Korea

Tel: +82-31-787-7164

Fax: +82-31-787-4097

E-mail:hyunsj@snu.ac.kr
A 10-year-old boy had a neuromuscular cervicothoracic kyphosis and kyphotic deformity got worse as he grew. He underwent posterior spinal fusion from T6 to pelvis two years ago. However, kyphosis progressed gradually, and difficulty occurred in breathing with a ventilator. We perform deformity correction with vertebral column resection at $\mathrm{T} 5$ and posterior fixation from $\mathrm{T} 2$ to $\mathrm{T} 9$ and posterior onlay fusion. Surgical correction is offered to stop the kyphosis progression, and finally to maintain the airway. After surgery, the patient transferred to an intensive care unit for respiratory care. The patient's breathing was much better than before surgery, and the patient was transferred to the general ward. We report the importance of postoperative care in spinal deformity patient with respiratory distress.

Keywords: Neuromuscular kyphosis; Spinal deformity surgery; Pulmonary complications; Intensive care

\section{INTRODUCTION}

Spinal deformity surgery in children with neuromuscular spinal deformity (NSD) is associated with a high morbidity rate ${ }^{1,4,8-10}$. Respiratory complications following spinal deformity surgery in patients with NSD have been reported from $15.6 \%$ to $28.2 \%^{2,4,8,9)}$. Respiratory complications may cause further impairment of respiratory function, leading to prolonged intubation time, and the need for re-intubation.

Postoperative intensive care may reduce postoperative morbidity and allow fast recovery. We report a case of severe neuromuscular cervicothoracic kyphosis treated with deformity correction and postoperative intensive care.

\section{CLINICAL PRESENTATION}

A 10-year-old boy had a neuromuscular cervicothoracic kyphosis. The kyphotic deformity became worse as he grew. He became paraplegic, with complete loss of bowel and bladder sensation. Therefore, he underwent posterior spinal fusion from $\mathrm{T} 6$ to pelvis two years ago. However, kyphosis progressed gradually, and his respiratory function also gradually worsened. He underwent tracheostomy and breathed using a ventilator. The patient was unable to eat enough food by mouth. So, the gastrostomy tube was inserted. However, kyphosis progressed gradually, and difficulty occurred in breathing with a ventilator (Fig. 1). The patient suffered from pneumonia repeatedly, and his nutrition gradually worsened.

Before surgery, the patient's pulmonary physician was contacted,

Copyright (C) 2019 The Korean Neurointensive Care Society

This is an Open Access article distributed under the terms of the Creative Commons Attribution Non-Commercial License (http://creativecommons.org/licenses/by-nc/4.0/) which permits unrestricted non-commercial use, distribution, and reproduction in any medium, provided the original work is properly cited. 
and bronchoscopy was done. There was an increased purulent secretion at the left lower lobe, but no other abnormal findings were observed. We perform deformity correction surgery. Under general anesthesia, the patient's head was fixed by using 3-pin head fixator. (Fig. 2A) Neck extension was attempted to correct cervicothoracic kyphosis, but the kyphosis was not corrected at all. After lamina exposure, pedicle screws were inserted from $\mathrm{T} 2$ to T9. Verte-
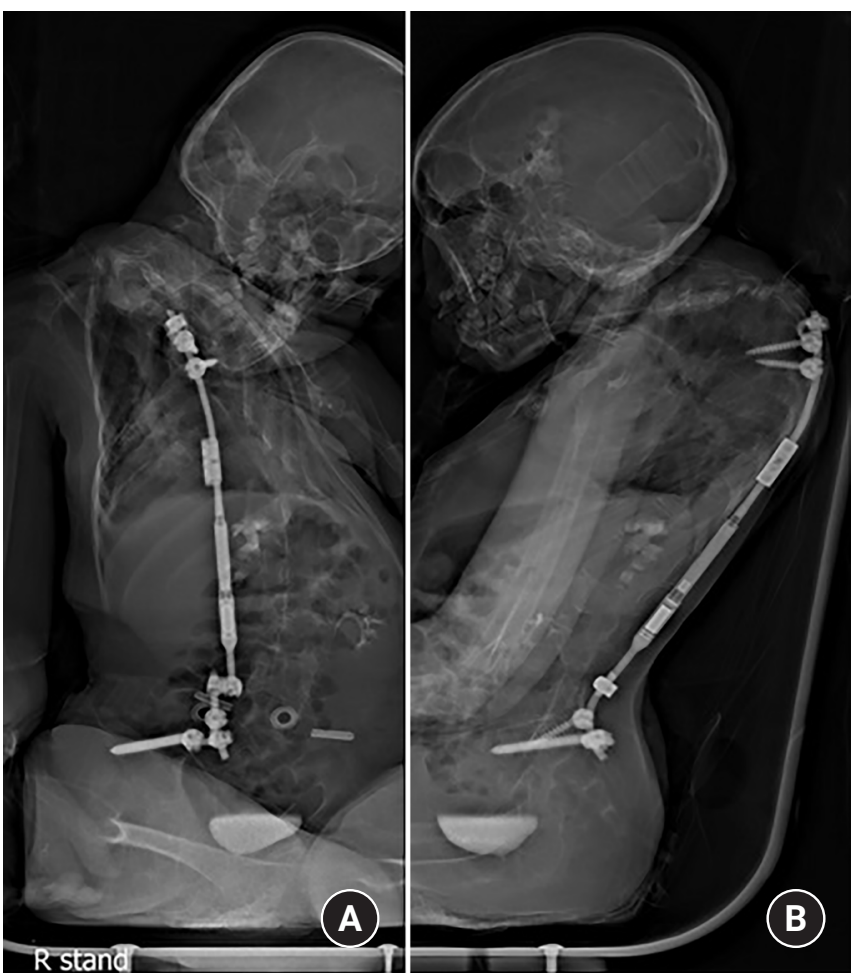

Fig. 1. (A) Preoperative whole spine anterior-posterior radiograph. (B) Preoperative whole spine lateral radiograph. bral column resection was done at the T5 level. After dekyphosis, pedicle screws were connected by a rod. The domino was used to connect the old rod with the new rod (Fig. 2B). Posterior onlay fusion was done using local bone, resected rib bone, allobone chip, demineralized bone matrix, and bone morphogenetic protein. During surgery, there was no significant change in intraoperative neuromonitoring.

After surgery, the patient transferred to an intensive care unit for respiratory care. Mechanical ventilation was applied for one day. Frequent suctioning and chest percussion, humidified oxygen were applied four times a day. Despite these aggressive efforts, fever $\left(38.6^{\circ} \mathrm{C}\right)$ occurred on postoperative two days. Chest X-ray showed no specific findings. However, prophylactic antibiotic (piperacillin/tazobactam) was administered. After that, no fever occurred. The patient was transferred to the general ward on postoperative three days. Whole spine $\mathrm{X}$-rays performed on postoperative seven days confirmed that deformity correction had been successfully achieved. (Fig. 3) The patient's breathing was much better than before surgery.

\section{DISCUSSION}

Morbidity following spinal deformity surgery for NSD is high, especially in respiratory complications ${ }^{1,48-10)}$. Therefore, perioperative intensive lung care is essential for improving surgical outcome. The previous study suggests a perioperative protocol for optimizing respiratory function ${ }^{7}$. Preoperatively, it is recommended to start regimen, including percussion vest therapy, cough assist, an increase in nebulizer treatments, and frequent suctioning. Postoperatively, patients without a history of pneumonia or a tracheostomy follow a protocol that includes humidified oxygen
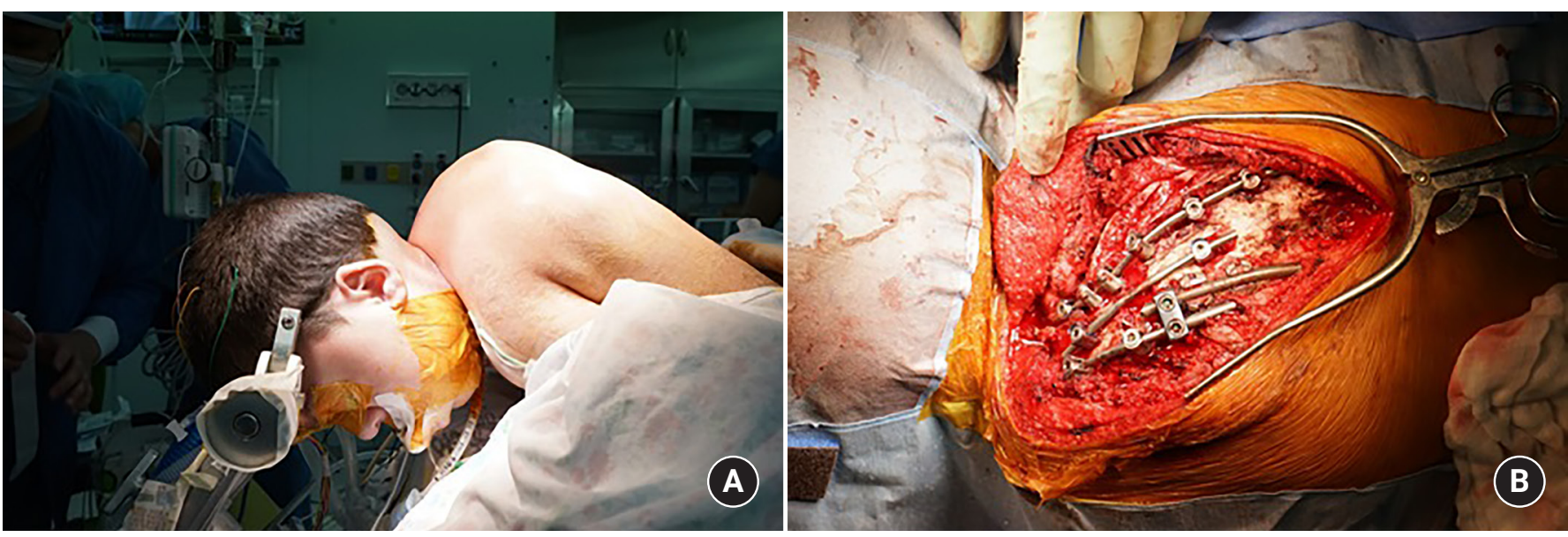

Fig. 2. (A) Intraoperative photographs were taken after head fixation. (B) Intraoperative photographs were taken after vertebral column resection at $\mathrm{T} 5$ level and posterior spinal fixation from $\mathrm{T} 2$ to $\mathrm{T} 9$. 


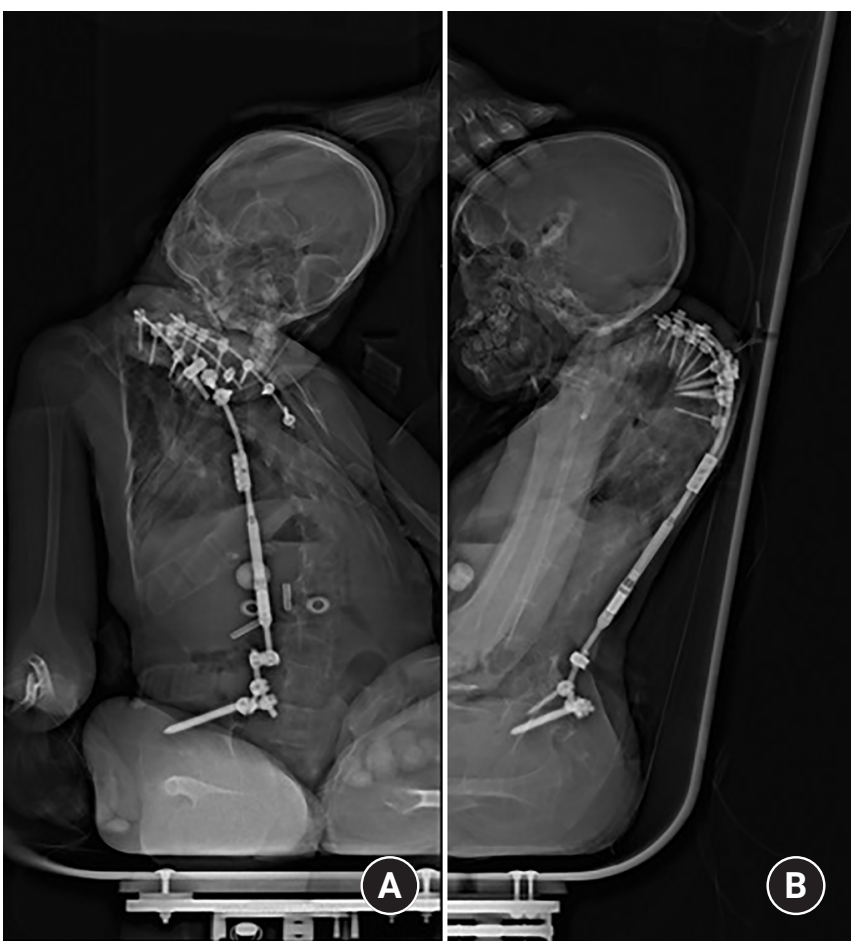

Fig. 3. (A) Postoperative whole spine anterior-posterior radiograph. (B) Postoperative whole spine lateral radiograph.

and nebulized albuterol treatment four times per day.

NSD patients with tracheostomies were more likely to experience respiratory complications, although these preventative and aggressive regimens were applied preoperatively and postoperatively. The presence of tracheostomy is associated with negative outcomes after treatment of various conditions ${ }^{3,5)}$. History of pneumonia is a clinically important independent predictor of increased risk of respiratory complications ${ }^{7}$. The association between tracheostomy presence and postoperative pneumonia has been reported ${ }^{3,6)}$. The previous study reported the association of gastrostomy tube and respiratory complications ${ }^{7}$. The presence of a gastrostomy was a risk factor of respiratory complications after spinal deformity surgery in NSD patients. The presence of tracheostomy, history of pneumonia, and the presence of gastrostomy may lead to worsened surgical outcomes.

In our case, surgical correction was offered to stop the deformity progression and to maintain the function of breathing. Before surgery, bronchoscopy was done to evaluate lung condition. After the operation, the patient was transferred to intensive care unit even though there were no respiratory events during the surgery. Frequent chest percussion and suctioning, cough assist, and nebulizer treatments were applied. However, the patient developed a fever $\left(38.6^{\circ} \mathrm{C}\right)$ on postoperative two days. Tazocin (piperacillin/tazobactam) was administered as a prophylactic antibiotic. Chest X-ray showed no specific findings, and pneumonia did not occur. Postoperative three days, the patient was transferred to a general ward. In this case, we performed aggressive lung care, including prophylactic antibiotics, before and after surgery. Given the rarity of this deformity, the optimal regimen for perioperative lung care remains unclear. However, respiratory complications can be reduced by active lung care after spinal deformity correction has been achieved in NSD patients.

\section{CONCLUSION}

A tracheostomy tube present or a history of pneumonia are risk factors of postoperative respiratory complications in NMD patients. We suggest that intensive lung care should be performed even if there were no respiratory events during the deformity correction surgery.

\section{NOTES}

\section{Conflict of interest}

No potential conflict of interest relevant to this article was reported.

\section{ACKNOWLEDGEMENTS}

None.

\section{REFERENCES}

1. Barsdorf AI, Sproule DM, Kaufmann P. Scoliosis surgery in children with neuromuscular disease: findings from the US National Inpatient Sample, 1997 to 2003. Arch Neurol 2010;67: 231-235.

2. Bendon AA, George KA, Patel D. Perioperative complications and outcomes in children with cerebral palsy undergoing scoliosis surgery. Paediatr Anaesth 2016;26:970-975.

3. Birkeland AC, Rosko AJ, Beesley L, Bellile E, Chinn SB, Shuman AG, et al. Preoperative Tracheostomy Is Associated with Poor Disease-Free Survival in Recurrent Laryngeal Cancer. Otolaryngol Head Neck Surg 2017; 157:432-438.

4. Brooks JT, Sponseller PD. What's New in the Management of Neuromuscular Scoliosis. J Pediatr Orthop 2016;36:627-633.

5. Hayashi T, Fujiwara Y, Sakai H, Maeda T, Ueta T, Shiba K. Risk factors for severe dysphagia in acute cervical spinal cord injury. Spinal Cord 2017;55:940-943.

6. Li L, Yuan W, Zhang S, Wang K, Ruan H. Analysis of Risk Factors for Pneumonia in 482 Patients Undergoing Oral Cancer 
Surgery With Tracheotomy. J Oral Maxillofac Surg 2016;74: 415-419.

7. Luhmann SJ, Furdock R. Preoperative Variables Associated With Respiratory Complications After Pediatric Neuromuscular Spine Deformity Surgery. Spine Deform 2019;7:107-111.

8. Pesenti S, Blondel B, Peltier E, Launay F, Fuentes S, Bollini G, et al. Experience in Perioperative Management of Patients Undergoing Posterior Spine Fusion for Neuromuscular Scoliosis. Biomed Res Int 2016;2016:3053056.
9. Rumalla K, Yarbrough CK, Pugely AJ, Koester L, Dorward IG. Spinal fusion for pediatric neuromuscular scoliosis: national trends, complications, and in-hospital outcomes. J Neurosurg Spine 2016;25:500-508.

10. Sharma S, Wu C, Andersen T, Wang Y, Hansen ES, Bunger CE. Prevalence of complications in neuromuscular scoliosis surgery: a literature meta-analysis from the past 15 years. Eur Spine J 2013;22:1230-1249. 